\title{
The discrepant epidemiology of Middle East respiratory syndrome coronavirus (MERS-CoV)
}

\author{
C. Raina MacIntyre
}

Published online: 25 July 2014

(c) The Author(s) 2014. This article is published with open access at Springerlink.com

\begin{abstract}
The Middle East respiratory syndrome coronavirus (MERS-CoV) is a newly emerged infection in humans affecting the Arabian Peninsula, Europe, and North Africa. The source and persistence of the infection in humans remains unknown. The aim of this paper was to apply a risk analysis approach to the epidemiology of MERS-CoV and to understand the source of ongoing infections. The epidemiology of MERS-CoV was reviewed and compared to SARS. Each observed feature of MERSCoV epidemiology was summarized and fitted to either an epidemic or one of two sporadic scenarios (either animal or deliberate release). As of May 2014, MERS-CoV has infected over 681 people and killed a further 204 over 2 years. In contrast, there were 8,273 cases and 775 deaths from SARS within 8 months. MERS-CoV has a more sporadic pattern unlike the clear epidemic pattern seen with SARS, and an unusual concentration of cases in the Middle East, without epidemics in other countries to which it has spread. SARS, with a higher reproductive number (R0), was eliminated from humans within 8 months of emerging, yet MERS-CoV, with a low R0 has persisted in humans over a far more prolonged period. This is at odds with the expected behavior of a virus with a low R0, which theoretically should not persist unless there are ongoing introductions of infection into humans, and poses the question "what is the source of continuing infections in humans?" A hospital outbreak in Al Ahsa, the Kingdom of Saudi Arabia (KSA), had a classic epidemic pattern with some humanto-human transmission. However, 3 different strains were
\end{abstract}

C. R. MacIntyre ( $\square)$

School of Public Health and Community Medicine,

Faculty of Medicine, University of New South Wales,

Sydney, NSW 2052, Australia

e-mail: r.macintyre@unsw.edu.au identified in that outbreak, an unexpected and unexplained finding for what appears to be a single source outbreak. Since this outbreak in April 2013, there has been a large increase in new cases, mainly in KSA in April and May 2014, with no corresponding epidemics in other countries. Yet MERS-CoV was present in KSA over several mass gatherings (which predispose to epidemics), including the Hajj pilgrimage, without an epidemic arising. Furthermore, although the virus has been identified in bats and camels, the mode of ongoing transmission to humans remains uncertain. Although some cases appear to be transmitted from human to human, and a few have animal or camel exposure, many cases have no history of contact with either animals or human cases. A high proportion of asymptomatic or otherwise undetected cases have been postulated as an explanation for the unusual epidemiology, yet active surveillance does not support this. When the observed data were fitted to different disease patterns, the features of MERS-CoV fit better with a sporadic pattern, with evidence for either deliberate release or an animal source. There are many discrepancies in the observed epidemiology of MERS-CoV, which better fits a sporadic than an epidemic pattern. Possible explanations of the unusual features of the epidemiology include human-to-human transmission with a large proportion of undetected cases; or sporadic ongoing infections from a non-human source; or a combination of both. Possible sources of ongoing sporadic infection in humans include animals (camels appear the most likely source), or deliberate release. The latter could explain 3 strains being present in a single hospital outbreak. Genetic testing should be conducted to determine whether the virus is evolving to be more transmissible. Better ascertainment of mild or asymptomatic cases is also needed. Finally, the discrepant epidemiology warrants critical analysis of all possible explanations, and 
involvement of all stakeholders in biosecurity, and deliberate release must be seriously considered and at least acknowledged as a possibility.

Keywords MERS-CoV - Emerging infectious disease . Epidemiology $\cdot$ Bioterrorism

\section{Background}

Viruses have the capacity to evolve, to exchange genetic material between strains that infect different species, and to emerge into new threats to human health. The Middle East Respiratory Syndrome Coronavirus (MERS-CoV) which causes severe acute respiratory illness with high mortality emerged in April 2012 in the Middle East and spread mainly through travel to several countries including the UK, Germany, France, Tunisia, Spain, Italy and the US (World Health Organization 2013). Comparisons have been made to SARS, a related coronavirus which displayed a classic epidemic pattern 10 years earlier. In just 8 months, SARS resulted in 8,273 cases and 775 deaths (World Health Organization). MERS-CoV in contrast to the clear epidemic pattern of SARS, has a different epidemiology, continuing to cause at least 681 non-fatal human infections and 204 deaths after two years, without a clear source (World Health Organization 2013; Wallinga and Teunis 2004). Unlike SARS, which spawned satellite epidemics in the countries to which it spread, the majority of cases of MERS have remained in the Middle East, mainly the Kingdom of Saudi Arabia (KSA), with no satellite epidemics or sustained human-to-human spread in other regions of the world, despite a large upsurge in cases in KSA in April and May 2014.

Control of emerging infectious diseases falls within the scope of biosecurity, which is a multidisciplinary field incorporating biomedical researchers, public health experts, emergency services, military, law enforcement, and risk analysts among others. This paper is intended for a broad, multidisciplinary readership, particularly for those in the non-health disciplines involved in biosecurity. The paper aims to apply a risk analysis approach to publicly available data on MERS, to shift the paradigms of thinking about emerging infectious diseases, to determine whether the current surveillance and response to MERS is asking the right questions, to consider whether MERS might be a category $\mathrm{C}$ bioterrorism agent, and to engage all relevant sectors in the response.

Infectious diseases are mainly classified as either epidemic, sporadic, or endemic (Anderson and May 2008). Epidemics are defined by a rapid rise in cases over time, not by the total number of cases. A small epidemic is usually termed an outbreak, and an epidemic which spreads globally is termed a pandemic. Diseases which have been present for long periods in a population but do not display a rapid rise in incidence over a short-time period, such as malaria, are classified as endemic, even with high case numbers. Diseases which have low case numbers and occur occasionally are classified as sporadic. MERS-CoV has displayed mixed features of both an epidemic and sporadic nature (World Health Organization 2013).

A classic epidemic curve is a normal distribution of cases over time (bell curve), showing a small number of first cases, a rapid rise in new cases over a short duration, an epidemic peak, and then a decline in cases. This corresponds to known mathematically predictable features of infectious diseases, which relate to human beings existing in mutually exclusive states of being infected, immune or susceptible to any infection (Anderson and May 2008). For any infection, the rate of transition of people within a given population from one state to another (e.g., susceptible to infected) can be modeled, using known parameters of the pathogen. At the beginning of an epidemic, there is a critical threshold of susceptible (non-immune) people in the population which gives rise to epidemic conditions, but at the peak, enough people in the population have been infected and are immune, thus stopping ongoing transmission. This then causes a decline in cases, giving rise to the tail end of an epidemic curve. Epidemics can be caused by humans becoming infected from either a single point source or by person-to-person transmission. A point source epidemic (such as food poisoning from a single contaminated food source consumed by many people) typically shows a unimodal epidemic curve which then dies down, whereas an epidemic with person-to-person transmission (such as influenza), may show a bimodal peak, representing the second generation of cases. Even in this case, with ongoing human-to-human transmission, eventually, when enough people are infected and become immune, the epidemic will die down and cease, until a critical threshold of susceptibility is again reached in the population, either through waning immunity, births, or migration (Anderson and May 2008).

SARS displayed all the features of an epidemic (and then a pandemic), with occurrence over a very short period of time, a rapid rise of cases from the time of onset of the first case, and classic epidemic curves and satellite epidemics in most countries which it affected, and then a decline in the epidemic (Wallinga and Teunis 2004). In the context of the well understood behavior of infectious diseases and the accepted science of epidemiology, the aim of this paper was to review the epidemiology of MERS-CoV, to compare it to SARS, and to explore all possible explanations for the observed epidemiologic data by fitting observed epidemiologic features to either epidemic or sporadic scenarios. 


\section{Methods}

A literature search was conducted for all available data on MERS-CoV, including MEDLINE, EMBASE, PubMed, and gray literature reports such as from the World Health Organization (WHO) and ProMED-mail, a rapid outbreak communication site. Publicly available surveillance data were used to compare total cases over total time for MERS$\mathrm{CoV}$ with a related coronavirus, SARS, and to compare the epidemiologic pattern of each virus.

The key features of the epidemiology of MERS-CoV were then analyzed in detail. Finally, a risk analysis approach was taken to classify each feature of the observed epidemiology of MERS-CoV as being consistent with either an epidemic or a sporadic pattern. An epidemic pattern would arise from sustained person-to-person transmission of infection and a R0 greater than 1 (Anderson and May 2008). A sporadic pattern would arise from some other (non-human) source of ongoing infection to humans, with generally a low R0 $(<1)$ which does not facilitate sustained person-to-person transmission. For completeness, possible non-human sources of ongoing human infections are a zoonotic or deliberate release. No judgement was made about whether deliberate release may be an engineered virus or a naturally occurring virus, nor was any judgment made about reasons or motives for such a scenario. The available data and evidence were simply assessed according to known epidemiologic principles of disease transmission. The publicly available data and evidence were categorized into discrete epidemiologic features, and then, each feature was fitted to the pattern which best explained it, either epidemic or sporadic. Given that a clear zoonotic origin of MERS-CoV explaining all sporadic cases has not been established, and a mixed pattern has been observed, two sporadic patterns were considered-a zoonotic source or deliberate release. $\mathrm{H} 5 \mathrm{~N} 1$ is a classic example of a sporadic human infection from a zoonotic source. In the case of $\mathrm{H} 5 \mathrm{~N} 1$ infections in humans, the vast majority of cases have clear exposure to a plausible zoonotic source known to host the same virus (Uyeki 2008). This has not been the case with MERS-CoV, which justifies considering other sporadic sources such as deliberate release.

\section{Results}

Figure 1 compares SARS and MERS-CoV, showing the number of total cases and a timeline of persistence in human populations since onset of the first case. This highlights the difference between SARS and MERS-CoV, showing the short time frame within which SARS persisted and was then eliminated in humans, and the large number of cases. MERS-CoV, in contrast, has a relatively low number of cases and has persisted for almost three times the duration, and at the time of writing this, continues to occur in humans.

\subsection{Comparison of features of SARS and MERS-CoV}

MERS-CoV first emerged in a hospital in Jordan in April 2012 (World Health Organization 2013), with later cases and clusters in KSA, Qatar, and the United Arab Emirates. Travel from these regions resulted in four additional clusters in Europe and North Africa, and more recently in the USA (World Health Organization 2013), with limited local transmission occurring in each site. Furthermore, several of the busiest airports in the world are located in countries where MERS-CoV has been transmitted, yet no epidemic has occurred in other countries as it did with SARS, which caused classic epidemics in almost all countries to which it spread, thereby displaying a consistency in epidemiologic pattern (Anderson and May 2008; Airports Council International 2012).

The majority of MERS-CoV cases have been associated with hospital outbreaks in Jordan, KSA, UAE and France. These clusters have been somewhat variable in clinical features, with the first outbreak in Jordon notably featuring renal failure, which does not feature as much in other clusters. SARS was also predominantly a nosocomial infection (Wallinga and Teunis 2004), but MERS$\mathrm{CoV}$ is unlike SARS. The median age of MERS-CoV is 51 years, and risk factors include male sex, immunosuppression, hospitalization and chronic disease (Centers for Disease Control and Prevention 2012). In contrast, SARS was characterized by younger age, more female cases and less co-morbidity. The male predominance of MERS-CoV may reflect a lower risk of exposure for Middle Eastern women due to wearing of Hijab, which covers the nose and mouth.

MERS-CoV did not display an epidemic peak in the first 12 months after emergence as did SARS (Wallinga and Teunis 2004). Rather, there was a sporadic pattern with persistence over a longer duration than expected for a disease with low estimated infectiousness (see Fig. 1). While there has been a large surge in cases in KSA in 2014, the pattern is still suggestive of a sporadic rather than epidemic source of ongoing infection in humans. The incubation period is estimated to be around 5.5 days (Cauchemez et al. 2014). The reproductive number, R0 is estimated to lie between 0.6 and 1.3 , which can be interpreted as MERS-CoV having low epidemic potential (Cauchemez et al. 2013; 2014; Breban et al. 2013). 
Fig. 1 Epidemiologic pattern from time of first reported caseSARS vs MERS-CoV. Data sources: World Health organisation http://www.who. int/csr/disease/coronavirus_ infections/en/ and http://www. who.int/csr/sars/country/en/

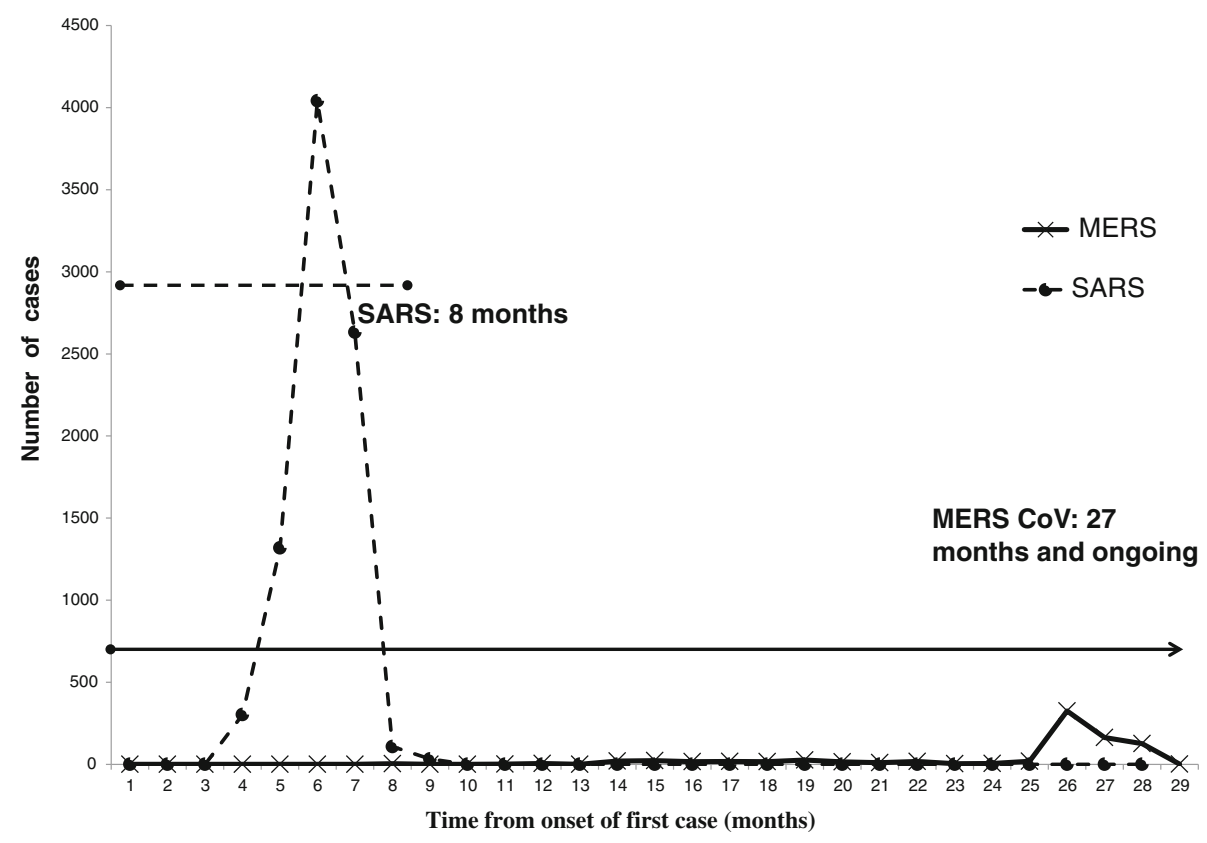

\subsection{Features of MERS-CoV}

In April 2013, an outbreak of 26 cases of MERS-CoV occurred in a hospital in Al Ahsa, KSA. This outbreak shows a typical short-duration epidemic curve suggestive of an infection with a R $0>1$, and consistent with a personto-person nosocomial outbreak caused by a single strain (Assiri et al. 2013). Curiously, phylogenetic analysis of the patient samples showed at least three different genotypes were present in this outbreak, and at least five of thirteen infections could not be explained by person-to-person transmission within the hospital (Cotten et al. 2013a). This suggests the hospital outbreak was caused by multiple independent introductions, along with some person-toperson transmission (Cotten et al. 2013a). The heterogenous transmission pattern in this outbreak is reminiscent of SARS, but multiple introductions into single outbreaks were not observed in nosocomial outbreaks of SARS. Further, the source of multiple introductions is unknown for MERS-CoV. It is possible that superspreading events may have a role in the epidemiology of MERS-CoV, but this does not explain the different genotypes present in the one outbreak. Figure 2 also shows that after more than a year of sporadic, infrequent cases that there has been a more sustained increase in cases since May 2013, mainly due to increased cases in KSA, with case numbers in other countries remaining low. Saudi Arabia is bordered by other countries and is a travel hub, yet this large increase in cases, at odds with the pattern seen from March 2012 until April 2013, has not been seen in other countries. If the large recent increase in cases in KSA is due largely to person-to-person transmission, other similar increases (satellite epidemics) would be expected in other countries (as was seen with SARS, with epidemics in each affected country), yet this consistency of global epidemiology has not been observed to date.

Travel and globalization make mass gatherings such as the Hajj pilgrimage a high risk for the spread of infectious diseases around the world. Another contradictory feature of MERS-CoV is that despite evidence of person-to-person transmission in some outbreaks such as Al Ahsa, no outbreaks arose from the Umrah or Hajj pilgrimages in KSA over two consecutive years, 2012 and 2013. More than four million pilgrims attend the Hajj alone, which is recognised as a risk for epidemics of infectious diseases (Memish and Rabeeah 2012). Active surveillance of symptomatic pilgrims in 2012 failed to detect MERS-CoV infections, and no cases were reported in returning pilgrims that year (Gautret et al. 2013). Furthermore, surveillance of Hajj pilgrims returning to high-income countries did not detect any cases in 2012 (World Health Organization 2013), nor were any antibodies detected in a serologic survey of blood donors and abattoir workers in KSA at the time (Aburizaiza et al. 2013). Rates of MERS-CoV in family and health care workers contacts are also low (Memish et al. 2014). Additionally, no cases have been reported by pilgrims who travelled to KSA to perform Umrah during July and August in either 2012 or 2013. Two unconfirmed cases in returning pilgrims to Spain and a case returning to India were reported following the 2013 Hajj; however, it is not yet know whether these cases acquired MERS-CoV during the Hajj or elsewhere in the Middle East during their travels (World Health Organization 2013). The absence of an epidemic despite highly conducive close contact conditions 
Fig. 2 Cases of MERS-CoV since 2012. Data source: World Health organisation http://www. who.int/csr/disease/ coronavirus_infections/en/

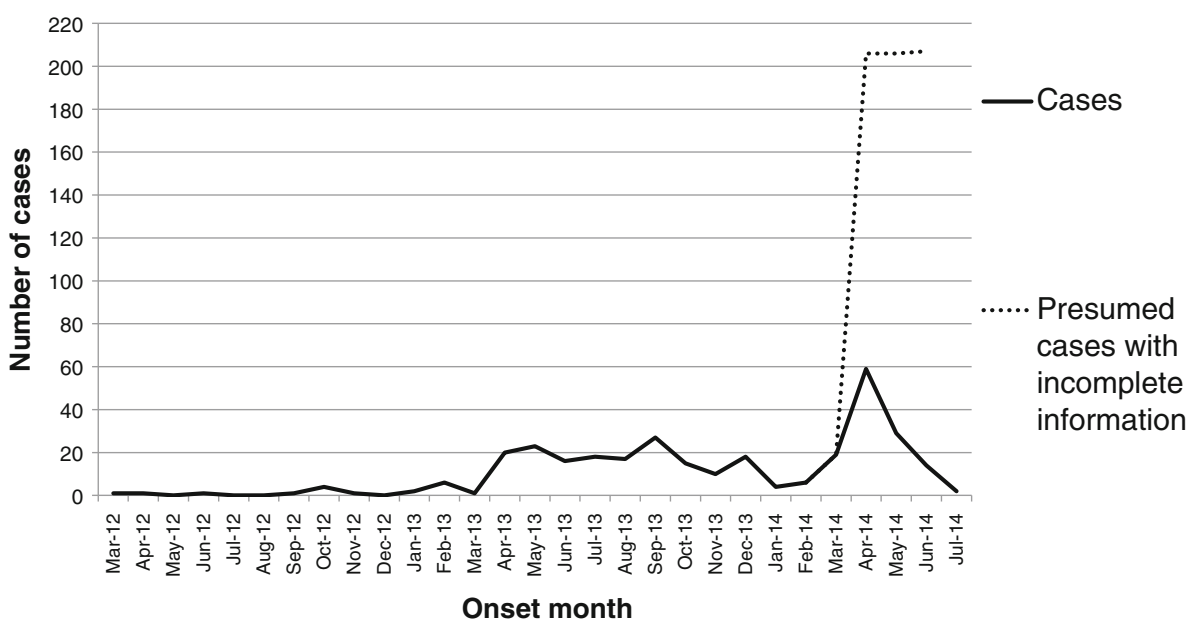

of four mass gatherings in KSA in 2012 and 2013, however, supports the sporadic pattern and estimated R $0<1$. Modelling shows that the probability of no secondary cases arising from the Hajj, if even one person with MERS was present at the time, is very low (Gardner et al. 2014).

MERS-CoV has persisted in the human population for at least three times as long as SARS, but with a lower R0, which in itself is contradictory. If the R0 is low, supported by current estimates and the lack of an epidemic arising from mass gatherings, then where are the infections coming from? The sources of ongoing transmission to humans can only be either person-to-person transmission, or sporadic transmission from a non-human source, or a combination of both. A non-human source could be zoonotic or deliberate release. While there is evidence of some person-toperson transmission (Assiri et al. 2013), this has not been shown to be the main mode of transmission. A zoonotic origin was identified for SARS, but spread was mainly person-to-person (Joseph et al. 2013). In contrast, personto-person spread cannot readily explain the persistence of MERS-CoV, but if animal exposure is the source, most cases have no consistent or clear history of animal contact. MERS-CoV has been identified in various bat species (Ithete et al. 2013; Cotten et al. 2013b; Annan et al. 2013). It has been identified in camels suggesting MERS-CoV or a related virus previously infected various camel populations, as early as 2003 (Reusken et al. 2013a; Perera et al. 2013; Group TWM-CR 2013; Meyer et al. 2014). While the MERS-CoV has been reportedly identified in camels of MERS-CoV patients, the actual mode and sequence of transmission remains unclear. Phylogenic analysis dates a common ancestor for all available MERS-CoV sequenced genomes to mid-2011 (Cotten et al. 2013b; Drosten et al. 2013), before the first confirmed case in Jordon. However, a cluster of viral sequences from the eastern Arabian Peninsula share a common ancestor estimated to be later in 2012, after the Jordan outbreak. It appears that MERS-CoV has infected humans through multiple introductions over a relatively short period of time, which resulted in limited clusters of cases. This corresponds to the observed epidemiology, although it is unknown how many introductions to humans may have occurred (Cauchemez et al. 2013).

One theory of MERS-CoV transmission is that a large number of asymptomatic or mild cases have gone undetected, thereby skewing the apparent epidemiology. A modelling study explains the epidemiology with substantial undetected mild cases, estimating that $62 \%$ of a total of 940 cases were undetected (Cauchemez et al. 2013).

Yet active screening and case finding efforts including contact tracing have not identified a substantial proportion of asymptomatic cases (Joseph et al. 2013). Only 5 out of 417 household and healthcare worker contacts became infected with MERS-CoV in the KSA cluster (Assiri et al 2013), and similarly, a serological study conducted on individuals from Jeddah and Makkah showed no evidence of widespread exposure to MERS-CoV (Aburizaiza et al. 2013). The model (Cauchemez et al. 2013) can only be fitted to the observed epidemiology of MERS-CoV if a large proportion of undetected cases are assumed to be true. Yet there is little evidence to support this. Further, if a large numbers of cases have been undetected, many of these would have arisen following mass gatherings such as the Hajj pilgrimage, where many pilgrims from highincome countries travel to and from KSA. While cases in returning international pilgrims may go undetected in lowincome countries with poor health systems, the likelihood of case detection is high in countries with advanced health systems and surveillance capacity. The lack of large numbers of cases in pilgrims returning to high-income countries following the Hajj in 2012 and 2013 does not support the hypothesis of a large proportion of undetected cases.

A summary of the various epidemiologic features of MERS Coronavirus and whether they support an epidemic 
Table 1 Supporting evidence of the epidemiologic pattern of MERS-CoV

\begin{tabular}{|c|c|c|c|}
\hline Supporting evidence of epidemiologic pattern & $\begin{array}{l}\text { Epidemic } \\
\text { (human to } \\
\text { human) }\end{array}$ & $\begin{array}{l}\text { Sporadic, } \\
\text { animal } \\
\text { source }\end{array}$ & $\begin{array}{l}\text { Sporadic, } \\
\text { deliberate } \\
\text { release }\end{array}$ \\
\hline Low case numbers for 12 months & & $\checkmark$ & $\boldsymbol{V}$ \\
\hline Low estimates of R0 & & $\checkmark$ & $\boldsymbol{\nu}$ \\
\hline Long persistence despite low R0 & & $\checkmark$ & $\boldsymbol{\nu}$ \\
\hline Some person-to-person transmission documented & $\checkmark$ & $?$ & $?$ \\
\hline Hospital outbreaks & $\checkmark$ & & $?^{\mathrm{a}}$ \\
\hline No epidemics arising from mass gatherings & & $\checkmark$ & $?$ \\
\hline Evidence of multiple introductions in a single outbreak & & & $\boldsymbol{\sim}$ \\
\hline Several cases without an identified epidemiologic link to a human case of MERS-CoV & & $\checkmark$ & $\boldsymbol{\sim}$ \\
\hline Several cases without an identified epidemiologic link to a zoonotic source & & $?$ & $\boldsymbol{\nu}$ \\
\hline Several cases with no link to human OR zoonotic source & & & $\boldsymbol{\sim}$ \\
\hline MERS-CoV identified in camels & & $\boldsymbol{\sim}$ & \\
\hline Multiple genetic strains in a single hospital outbreak at Al Ahsa Hospital & & $?^{\mathrm{b}}$ & $\boldsymbol{\sim}$ \\
\hline Active surveillance had not found evidence of a high proportion of undetected cases & & $\checkmark$ & $\boldsymbol{\nu}$ \\
\hline
\end{tabular}

${ }^{a}$ Deliberate release inside hospitals could explain this

${ }^{b}$ For this to be the explanation, simultaneous animal exposure would have to have occurred to animal hosts carrying different strains in the same time frame to cause concurrent human infections from different sources

or one of two sporadic patterns are shown in Table 1. This shows that the evidence supports a sporadic pattern, with slightly more weight to deliberate release compared to an animal source.

\section{Discussion}

There are still uncertainties and contradictions about the epidemiology of MERS-CoV, which is clearly different to SARS, fitting a more sporadic pattern even accounting for the recent surge in cases. SARS, in contrast had a clear epidemic pattern, including in countries to which it spread. The analysis of the features of MERS-CoV suggests that the source of ongoing sporadic infections could equally be due to an animal source or to deliberate release. The finding of MERS-CoV in camels does not rule out deliberate release, as a virus which is trophic to a host will, when present in an ecosystem, find that host. The WHO is investigating animal sources of infection as well as the mode of transmission to humans (Aburizaiza et al. 2013). Animal samples collected from KSA are currently undergoing analysis in US laboratories to help and identify the underlying zoonotic source(s). Additionally, the similarity between MERS-CoV and the closest bat coronaviruses suggests animal surveillance in South Africa and certain parts of Europe would also be valuable (Cotten et al. 2013b). Person-to-person transmission as the main mode of spread could only be explained if there is a large proportion of undetected mild or asymptomatic cases. This possibility is not supported by surveillance studies, but better screening methods could be developed. Serology for the detection of specific MERS$\mathrm{CoV}$ antibodies has been recently developed and validated with a limited number of specimens (Reusken et al. 2013b), but still need to be validated for use in the Arabian Peninsula and Jordan (Nicoll 2013).

The available data do not clearly fit a specific epidemiologic pattern, so it is important to think of all possible explanations, and not simply force the data into a known paradigm such as SARS. The possibility of deliberate release, which has not yet been discussed in the scientific literature, must be included for completeness, as this could explain the long persistence, the paradox of hospital outbreaks and yet no outbreaks following major mass gatherings, the evidence of multiple introductions, and the presence of multiple genotypes simultaneously in what appears on the surface to be a single hospital outbreak in $\mathrm{Al}$ Ahsa. This outbreak is the most puzzling to date because of the presence of multiple different genotypes in the outbreak within a very short time frame-this means that the patients with MERS-CoV had simultaneous or contemporaneous sporadic exposures (which have yet to be identified), either inside or outside the hospital. The published work to date has focused on selected parts of the epidemiology which can be explained, but no one to date has questioned this single unusual finding, and deliberate release, which is certainly a possible explanation, has not thus far been publicly debated.

It seems likely that MERS-CoV has persisted in human populations despite a low R0 due to a combination of 
sporadic transmission from a non-human source and some human-to-human transmission. The epidemiology appears to have changed somewhat from May 2013 onward, but there is no explanation for this change, which is due largely by an increase in KSA alone, nor evidence as yet that the virus is becoming more transmissible. The continued increase in cases in KSA in 2014, without satellite epidemics in other countries, and without consistent animal contact in all cases is inexplicable. Good diagnostic serological methods, serological surveys, contact tracing and other surveillance in affected areas are needed to quantify asymptomatic or mild infection and to identify exposures to other (non-human) sources of infection. The serological tools recently developed (Reusken et al. 2013b) can aid in large-scale contact studies. An epidemiological protocol to assess MERS-CoV cases in the UK was proposed, and related protocols to implement surveillance schemes among healthcare personnel, contacts of known and probable cases and the general population have been published by CONCISE (2013). These protocols will help quantify clinical characteristics (e.g., incubation and infectious period) and epidemiological characteristics (e.g., source, risk factors, R) of MERS-CoV. Open access to surveillance and screening data will assist in further understanding the disease (Palm et al. 2012).

\section{Conclusions}

In conclusion, a careful review of the paradoxes and inconsistencies in the epidemiology of MERS-CoV raises deliberate release as a possible explanation. The discrepant, varying epidemiology is consistent with deliberate release, as such an act cannot easily simulate expected natural patterns, and is likely to produce an inconsistent epidemiologic picture. When a new infectious disease emerges, bioterrorism, unless it is caused by an eradicated disease such as smallpox, may not be easily recognized for what it is unless we consider the possibility. A sporadic animal source of ongoing infections in humans is also possible, with most evidence supporting camels. However, the large increase in cases in 2014 without a clear accompanying history of camel contact in the majority of cases does not support this explanation. Other zoonotic infections which occur sporadically in humans such as $\mathrm{H} 5 \mathrm{~N} 1$ influenza show a far more consistent and clear history of contact with animals/birds. The virus may be emerging to be more transmissible, but there is no evidence for a clearly epidemic pattern as yet.

Control efforts to date been structured around an underlying assumption of a natural cause of this disease and has followed a standard public health response framework using knowledge of SARS as a guide (Perl et al.
2014). However, the epidemiologic features of MERS-Cov are clearly very different to SARS. There is a pressing need for inter-sectoral collaboration, for involvement of all stakeholders in biosecurity, including law enforcement and military, and for reflecting on the available data and interpreting it objectively, instead of trying to force the data into a paradigm created around the past SARS experience. While both are coronaviruses, their epidemiology is very different, and control of MERS coronavirus can only be achieved when we gain understanding of the observed paradoxes.

Conflict of interest The author declares there is no conflict of interest.

Open Access This article is distributed under the terms of the Creative Commons Attribution License which permits any use, distribution, and reproduction in any medium, provided the original author(s) and the source are credited.

\section{References}

Aburizaiza AS, Mattes FM, Azhar EI, Hassan AM, Memish ZA, Muth $\mathrm{D}$ et al (2014) Investigation of anti-MERS-Coronavirus antibodies in blood donors and abbatoir workers in Jeddah and Makkah, Kingdom of Saudi Arabia, Fall 2012. J Infect Dis 209:243-246

Airports Council International (2013) Preliminary 2012 world airport traffic and rankings. Available from: http://www.aci.aero

Anderson R, May R (2008) Infectious diseases of humans. Dynamics and control. Oxford University Press Inc., New York

Annan A, Baldwin H, Corman V, Klose S, Owusu M, Nkrumah EE et al (2013) Human betacoronavirus 2c EMC/2012-related viruses in bats, Ghana and Europe. Emerg Infect Dis 19(3):456-459

Assiri A, et al (2013) Hospital outbreak of Middle East respiratory syndrome coronavirus. New Engl J Med http://www.nejm.org/ doi/pdf/10.1056/NEJMoa1306742

Breban R, Riou J, Fontanet A (2013) Interhuman transmissibility of Middle East respiratory syndrome coronavirus: estimation of pandemic risk. Lancet http://ac.els-cdn.com/S01406736136149 20/1-s2.0-S0140673613614920-main.pdf?.tid=7c7bd8ce-ecf311e2-a34f-00000aacb360\&acdnat $=1373854308 \_04 b c 4 d c 5863$ $770 \mathrm{~b} 1711 \mathrm{ff} 1 \mathrm{ed} 88 \mathrm{~b} 0 \mathrm{fdd} 0$

Cauchemez S, Van Kerkhove MD, Riley S, Donnelly CA, Fraser C, Ferguson NM (2013) Transmission scenarios for Middle East respiratory syndrome coronavirus (MERS-CoV) and how to tell them apart. Euro Surveill 18(24):pii=20503

Cauchemez S, Fraser C, Van Kerkhove MD, Donnelly CA, Riley S, Rambaut A (2014) Middle East respiratory syndrome coronavirus: quantification of the extent of the epidemic, surveillance biases, and transmissibility. Lancet Infect Dis 14:50-56

Centers for Disease Control and Prevention (2013) Update: severe respiratory illness associated with Middle East respiratory syndrome coronavirus (MERS-CoV)—worldwide, 2012-2013. MMWR Morb Mortal Wkly Rep 62:1-4

CONSISE (2013) Novel coronavirus (MERSCoV). The consortium for the standardization of influenza seroepidemiology (CONSISE) Steering Committee 2013; http://consise.tghn.org/articles/ novel-coronavirus-ncov

Cotten M, Lam T, Watson SJ, Palser AL, Petrova V, Grant P, et al (2013a) Full-genome deep sequencing and phylogenetic analysis 
of novel human betacoronavirus. Emerg Infect Dis. doi:10.3201/ eid1905.130057

Cotten M et al (2013b) Transmission and evolution of the Middle East respiratory syndrome coronavirus in Saudi Arabia: a descriptive genomic study. Lancet 382:1993-2002

Drosten C, Seilmaier M, Corman V, Hartmann W, Scheible G, Sack S, Guggemos W et al (2013) Clinical features and virological analysis of a case of Middle East respiratory syndrome coronavirus infection. Lancet Infect Dis 13:745-751

Gardner L et al (2014) A scenario-based evaluation of the Middle East respiratory syndrome coronavirus and the Hajj. Risk Anal. doi:10.1111/risa.12253

Gautret P et al (2013) Lack of nasal carriage of novel corona virus (HCoV-EMC) in French Hajj pilgrims returning from the Hajj 2012, despite a high rate of respiratory symptoms. Clin Microbiol Infect 19(7):E315-E317

Group TWM-CR (2013) State of knowledge and data gaps of Middle East respiratory syndrome coronavirus (MERSCoV) in humans. PLOS Current Outbreaks

Ithete NL, Stoffberg S, Corman VM, Cottontail VM, Richards LR et al (2013) Close relative of human Middle East respiratory syndrome coronavirus in bat, South Africa. Emerg Infect Dis 19:1697-1699

Joseph C et al (2013) Highlights and conclusions from the technical consultative meeting on novel coronavirus infection, Cairo, Egypt, 14-16 January 2013. East Mediterr Health J 19(Suppl 1):S68-S74

Memish ZA, et al (2014) Screening for Middle East respiratory syndrome coronavirus infection in hospital patients and their healthcare worker and family contacts: a prospective descriptive study. Clin Microbiol Infect. doi:10.1111/1469-0691.12562

Memish Z, Rabeeah A (2012) Health conditions for travellers to Saudi Arabia for the Umrah and Hajj pilgrimage to Mecca: requirements for 2012 (1433). J Infect Public Health 5(2): 113-115

Meyer B, Müller MA, Corman VM, Reusken CB, Ritz D, Godeke G-D et al (2014) Antibodies against MERS coronavirus in dromedary camels, United Arab Emirates, 2003 and 2013. Emerg Infect Dis 20(4):552-559

Nicoll A (2013) Public health investigations required for protecting the population against novel coronaviruses. Eastern Mediterranean Health Journal

Palm D, et al (2012) Laboratory capability for molecular detection and confirmation of novel coronavirus in Europe, November 2012. Euro Surveill 17(49):pii=20335. http://www.eurosurveil lance.org/ViewArticle.aspx?ArticleId=20335

Perera R, Wang P, Gomaa M, El-Shesheny R, Kandeil A, Bagato O et al (2013) Seroepidemiology for MERS coronavirus using microneutralisation and pseudoparticle virus neutralisation assays reveal a high prevalence of antibody in dromedary camels in Egypt. Euro Surveill 18(36):pii=20574

Perl T, McGeer A, Price C (2014) Medusa's ugly head again: from SARS to MERS-CoV. Ann Intern Med 160(6):p432

Reusken CB, Haagmans BL, Müller MA, Gutierrez C, Godeke GJ, Meyer B et al (2013a) Middle East respiratory syndrome coronavirus neutralizing serum antibodies in dromedary camels: a comparative serological study. Lancet Infect Dis 13:859-866

Reusken C, Mou H, Godeke GJ, van der Hoek L, Meyer B, Müller MA et al (2013b) Specific serology for emerging human coronaviruses by protein microarray. Euro Surveill 18(14): pii $=20441$

Uyeki T (2008) Global epidemiology of human infections with highly pathogenic avian influenza A (H5N1) viruses. Respirology 13: S2-S9

Wallinga J, Teunis P (2004) Different epidemic curves for severe acute respiratory syndrome reveal similar impacts of control measures. Am J Epidemiol 160(6):509-516

World Health Organization (2013) Background and summary of novel coronavirus infection—as of 22 November 2013. http://www.who. int/csr/disease/coronavirus_infections/Update12_MERSCoV_ update_22Nov13.pdf

World Health Organization. Global alert and response (GAR): pandemic and epidemic diseases (http://www.who.int/csr/dis ease/en/) 